\title{
Apendicitis epiploica. Informe de un caso y revisión de la literatura
}

\author{
Dra. Angélica León-Hernández, ${ }^{*}$ Dr. José Martín Palacios-Acosta, ${ }^{* *}$ Dr. José Ignacio Guzmán-Mejía, ${ }^{* * *}$ \\ Dr. Jaime Shalkow-Klincovstein ${ }^{* * * *}$
}

\section{RESUMEN}

La apendicitis epiploica es una consecuencia de la torsión o trombosis espontánea de las venas de drenaje de los apéndices epiploicos en la serosa del colon. Esta afección puede simular múltiples procesos de abdomen agudo quirúrgico. Es una entidad extremadamente rara en edad pediátrica.

Se describe el caso de un escolar de diez años de edad con datos clínicos sugestivos de apendicitis aguda, que fue sometido a una intervención quirúrgica, durante la cual se hallaron prolongaciones de la grasa normal del colon, pedunculadas, adheridas a la serosa del ciego, que requirieron disección y resección; además se realizó una apendicectomía profiláctica. El paciente evolucionó favorablemente.

Palabras clave: Apendicitis epiploica, abdomen agudo, tomografía computarizada, apendicectomía.

\section{ABSTRACT}

Epiploic appendicitis is caused by torsion or spontaneous thrombosis of the draining veins of the epiploic appendages. This condition can mimick a variety of acute surgical abdomen. It is an extremely rare entity in childhood.

We present the case of a 10 year old boy, with clinical signs of acute appendicitis. Surgery was performed. During an exploratory laparotomy, several blind peduncular fatty lesions were identified adhering to the cecum's serosa, which were dissected and resected. A prophylactic appendectomy was performed. The patient had a favorable outcome.

Key words: Epiploic appendicitis, acute abdomen, computed tomography, appendectomy.

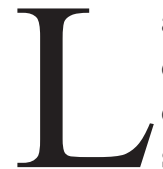

a apendicitis epiploica (AE) es una causa rara de abdomen agudo en la edad pediátrica. ${ }^{1,2} \mathrm{Se}$ debe a la torsión de apéndices epiploicos, que se acompaña de oclusión vascular, isquemia y trombosis venosa y arterial. Esta afección puede simular un abdomen agudo quirúrgico y conducir a una intervención quirúrgica no justificada. ${ }^{3,4}$

* Médico pasante del Servicio Social en Investigación

** Médico adscrito Servicio de Cirugía Oncológica. drjpalacios@hotmail.com

*** Medico adscrito Cirugía Oncológica. jignacioguzmanm@yahoo.com.mx

**** Jefe del Servicio de Cirugía Oncológica. drshalkow@yahoo.com

Correspondencia: Dra. Angélica León-Hernández. Instituto Nacional de Pediatría. Av. Insurgentes Sur 3700-C, Colonia Insurgentes Cuicuilco, C.P. 04530, Delegación Coyoacán, México D.F. angie_atl@hotmail.com

Recibido: septiembre, 2009. Aceptado: noviembre, 2009.

Este artículo debe citarse: León-Hernández A, Palacios-Acosta JM, Guzmán-Mejía Jl y col. Apendicitis epiploica. Informe de un caso y revisión de la literatura. Acta Pediatr Mex 2010;31(1):6-10.

La versión completa de este artículo también está disponible en: www.nietoeditores.com.mx
Se han publicado pocos casos. La baja frecuencia explica que rara vez se sospeche esta entidad clínicamente, por lo que su diagnóstico suele hacerse durante una intervención quirúrgica. ${ }^{5}$ No es infrecuente que el clínico piense en apendicitis aguda en casos de AE cecal, o bien que confunda la entidad con diverticulitis o con un problema ovárico, en casos de afección de colon ascendente o descendente. ${ }^{6,7}$

Clínicamente los pacientes refieren dolor abdominal localizado en fosa iliaca izquierda, acompañado de febrícula. ${ }^{8,9}$

Los estudios de laboratorio, la radiografía simple de abdomen y el ultrasonido son inespecíficos. La tomografía computarizada es el método de elección para el diagnóstico. Sin embargo, en la mayoría de los casos, el diagnóstico se realiza durante el acto quirúrgico. ${ }^{10,3}$

Actualmente, el tratamiento es fundamentalmente médico y consiste en medidas generales, reposo y analgésicos. ${ }^{17}$ El tratamiento quirúrgico se reserva para los casos en los que fracasa el tratamiento conservador o en los que haya complicaciones. ${ }^{19,22}$ 


\section{CASO CLÍNICO}

Niño de 10 años de edad, que acude a urgencias por presentar dolor cólico abdominal en la fosa ilíaca derecha, de tres días de evolución, acompañado de fiebre de $38^{\circ} \mathrm{C}$ y náusea. A la exploración física se encuentra conciente, hidratado, pálido ++ , con facies de dolor; orofaringe hiperemica; cardiopulmonar sin compromiso. Abdomen blando, depresible, doloroso a la palpación de la fosa iliaca derecha; rebote positivo. Biometría hemática: hemoglobina, $13.8 \mathrm{~g} / \mathrm{dL}$; hematócrito, $43.8 \%$; plaquetas 285,000 ; leucocitos 12,200, linfocitos $11.4 \%$, neutrófilos $80.4 \%$. Química sanguínea: glucosa $107 \mathrm{mg} / \mathrm{dL}$, creatinina 0.7 $\mathrm{mg} / \mathrm{dL}$, tiempo de protrombina 13.1 segundos; $74.6 \%$ de actividad. La radiografía simple de abdomen muestra escoliosis y borramiento del psoas. (Figura 1) Un ultrasonido abdominal de fosa ilíaca derecha mostró líquido libre de aproximadamente $10 \mathrm{~mL}$, con hipomotilidad del íleo terminal y abundante gas que no permitía visualizar el apéndice. El informe del estudio fue: proceso inflamatorio en la fosa iliaca derecha por probable apendicitis. (Figura 2)

Se realizó una laparotomía exploradora de urgencia; se halló una prolongación de grasa, pedunculada en el ciego

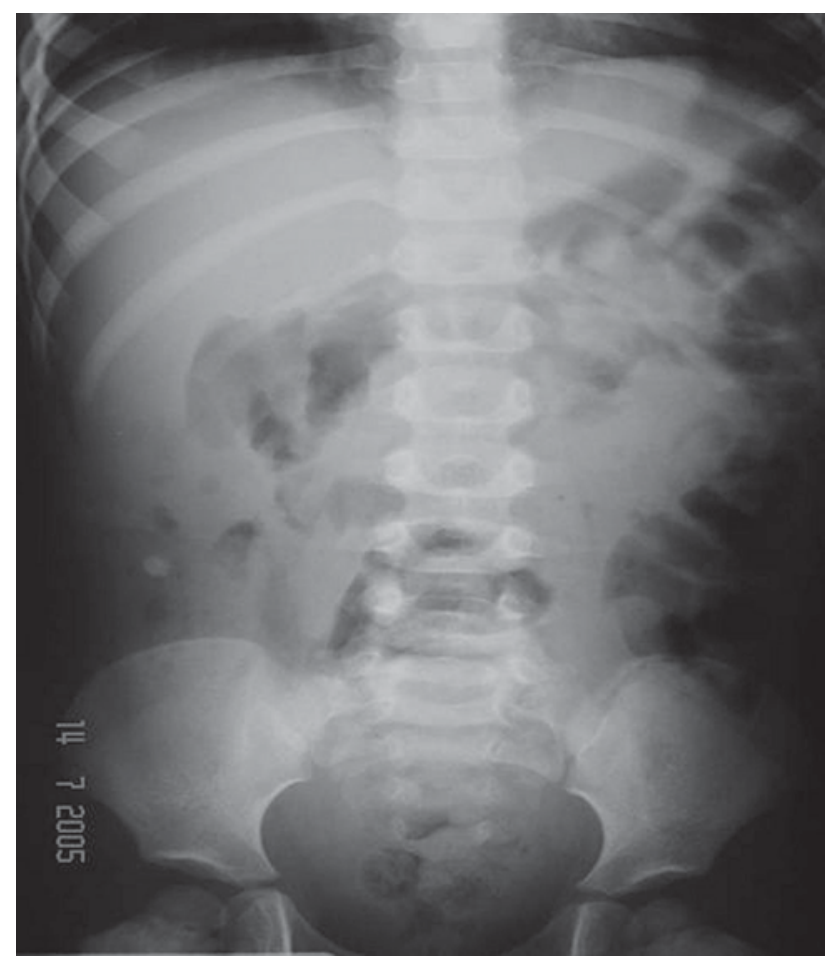

Figura 1. Radiografía simple de abdomen. Muestra escoliosis y borramiento del psoas.

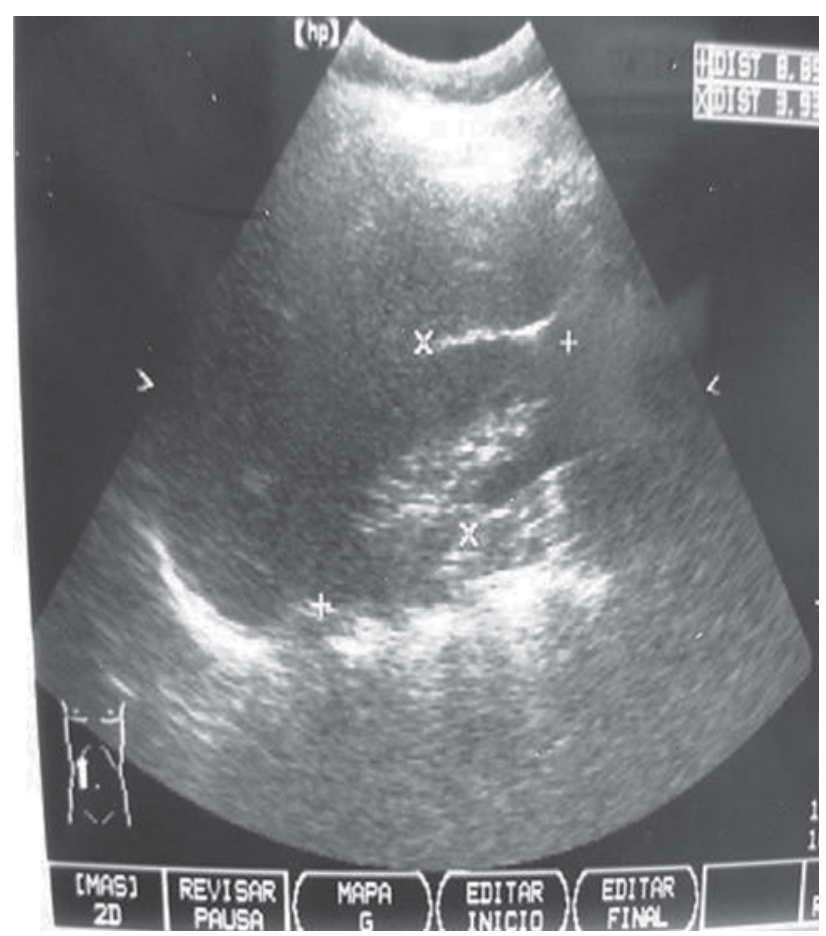

Figura 2. Ultrasonido abdominal de fosa iliaca derecha. Se observa líquido libre de aproximadamente $10 \mathrm{~mL}$ con hipomotilidad de íleo Terminal y abundante gas que no permite ver el apéndice cecal.

adherida a la serosa del colon, (Figura 3 ) $4 \mathrm{~cm}$ de largo y $2 \mathrm{~cm}$ de grosor aproximadamente, se disecó y se hizo ligadura de dicha prolongación. (Figura 4) Se hizo una apendicetomía profiláctica. (Figura 5)

El paciente evolucionó favorablemente en el postoperatorio; toleró alimentos por la vía oral a las 24 horas y egresó a las 48 horas en buenas condiciones.

El informe patológico fue: apendicitis epiploica y apéndice cecal sano.

\section{DISCUSIÓN}

La apendicitis epiploica es un proceso inflamatorio benigno y autolimitado del tejido adiposo intraabdominal; clínicamente causa cuadros de abdomen agudo que pueden requerir una intervención quirúrgica. ${ }^{1,5,9}$

En 1543 Vesalius describió por primera vez los “apéndices epiploicos". Vittre en 1703 informó el caso un apéndice epiploico libre, como un cuerpo extraño en la cavidad peritoneal. ${ }^{2,5}$

El término apendicitis epiploica fue acuñado por Lynn en $1956 .{ }^{3}$ En 1986 citado por 4 , se mostró por primera vez la apariencia de la apendicitis epiplóica. ${ }^{4}$ 


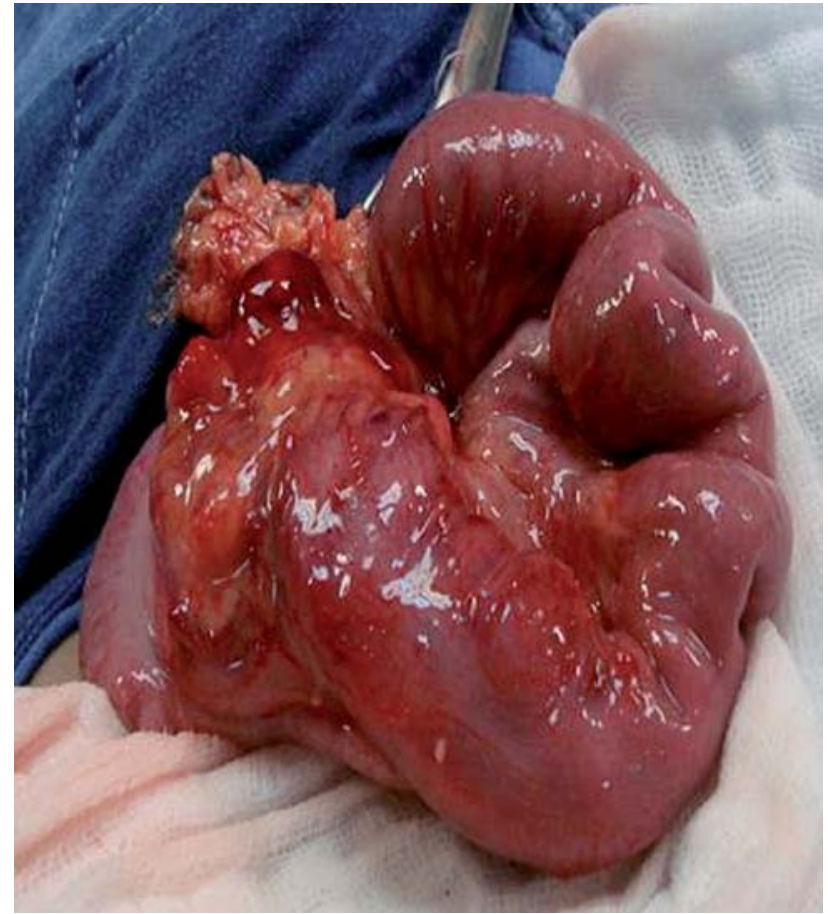

Figura 3. Prolongación de grasa, pedunculada en ciego adherida a la serosa del colon.

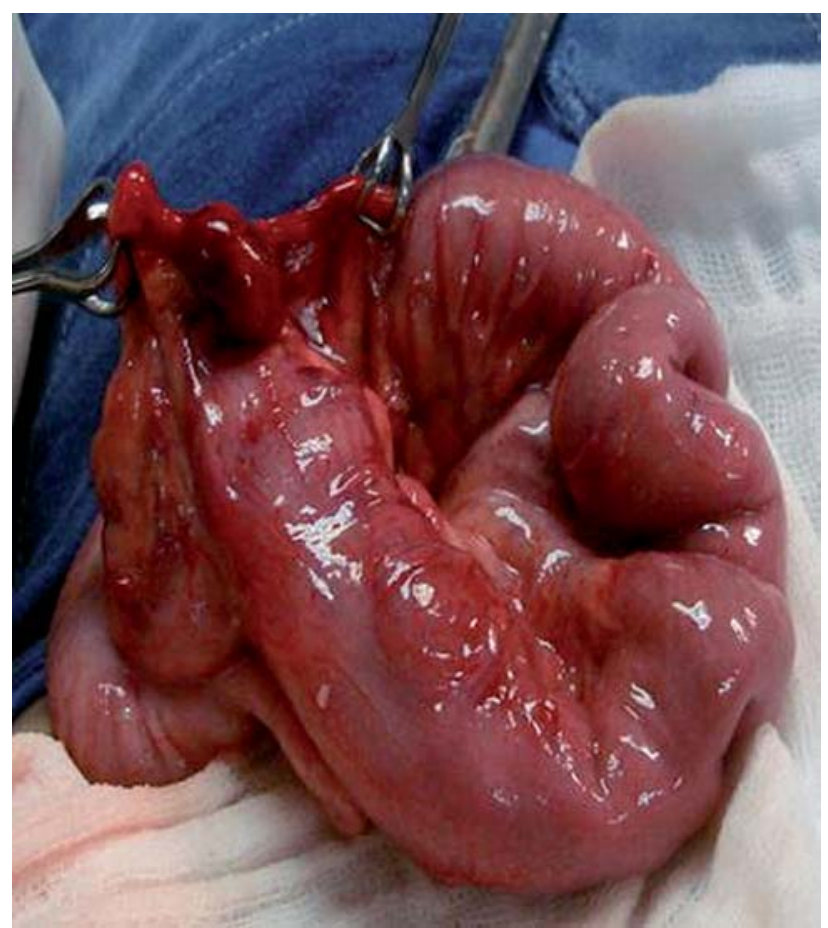

Figura 4. Disección ligadura del apéndice epiploico.

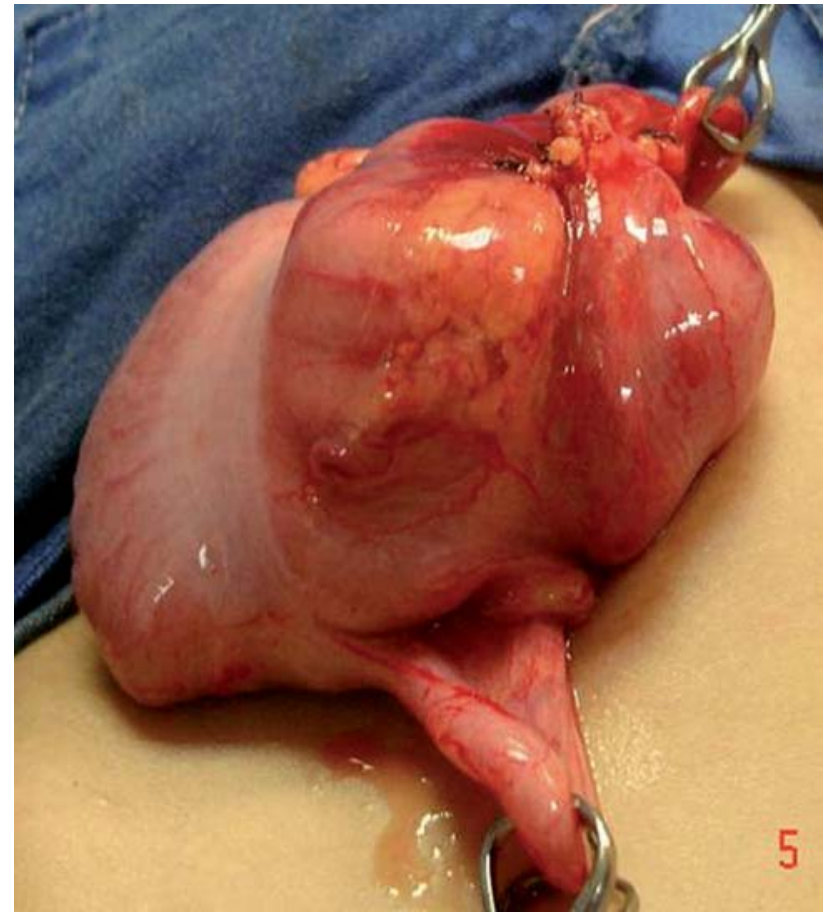

Figura 5. Apendicectomía.

Es una entidad infrecuente en niños. Se ve más entre la segunda y quinta décadas de la vida y es más frecuente en hombres $(2: 1) .{ }^{6,9,12}$

Los apéndices epiploicos son estructuras pedunculadas de tejido adiposo que protruyen de la superficie serosa del colon a la cavidad peritoneal. Están dispuestos en dos filas longitudinales separadas que se extienden desde el ciego hasta la unión rectosigmoidea. ${ }^{7,8}$

Existen entre 50 y 150 apéndices de este tipo: miden 2 a $5 \mathrm{~cm}$ de largo y 1 a $2 \mathrm{~cm}$ de grosor; son más numerosos y de mayor volumen los que se encuentran en el colon descendente y en el sigmoides. ${ }^{2,7}$ Se desconoce su función exacta. Se postulan varías teorías, entre ellas, que pueden servir como apoyo a las paredes del colon durante la peristalsis; que son un reservorio de sangre cuando el colon y sus vasos intramurales están contraídos; que representan un depósito energético y como epiplón en caso de procesos inflamatorios. ${ }^{9,10,18}$

Cada apéndice epiploico es irrigado por una o dos pequeñas arteriolas, ramas de los vasos rectos del colon. Drenan por venas únicas tortuosas que pasan a través de un pedículo estrecho. ${ }^{1,12 \text {, }}$

La forma pediculada, su gran movilidad y el hecho de tener irrigación Terminal, los hacen propensos a la 
torsión y a la trombosis venosa, lo que conduce a un infarto isquémico o hemorrágico y a la irritación peritoneal, que ocasiona un abdomen agudo, que simula un cuadro quirúrgico. ${ }^{9,11}$

Existen factores de riesgo para esta entidad: la obesidad, las hernias, el ejercicio intenso, las comidas abundantes. Cualquiera de éstas puede causar una ectasia venosa esplácnica y trombosis. ${ }^{12,15}$

El cuadro clínico es el de dolor abdominal intenso, de inicio súbito, localizado en fosa iliaca izquierda; acompañado en algunos casos de náusea o vómito y febrícula. $13,15,1$

Los exámenes de laboratorio pueden mostrar leucocitosis leve como en nuestro caso y velocidad de sedimentación elevada en las primeras horas, o aumento de la proteína $\mathrm{C}$ reactiva. ${ }^{16,14,3}$

El diagnóstico se confirma con estudios de imagen orientados fundamentalmente a descartar causas de abdomen agudo. ${ }^{1}$ El ultrasonido puede ser diagnóstico mostrando una masa ecogénica, no compresible, redonda u oval que coincide con el punto máximo del dolor. Este hallazgo es característico y se ha llamado "infarto graso focal". Sin embargo, es menos frecuente en pacientes obesos o localizaciones profundas. La utilidad del estudio depende por lo demás, de la habilidad de quien lo realiza. 9,19,22

El estudio de elección es la tomografía computarizada, que según algunos autores, muestra una apariencia patognomónica: ${ }^{17}$ una imagen hipodensa, redondeada u ovoide de densidad grasa (de -30 unidades Hansfield que corresponde a grasa) ubicada en la serosa de algún segmento del colon y el peritoneo parietal, limitada por un anillo hiperdenso de $2 \mathrm{~mm}$ de grosor que corresponde al halo hipogénico (signo del anillo característico del apéndice epiploico). ${ }^{10,12,13}$

En ocasiones se visualizan finas líneas densas en el centro de la lesión, que representan los vasos trombosados del apéndice epiplóico. Un signo clave es la identificación del proceso inflamatorio de la pared del colon. ${ }^{18,19}$

En pacientes tratados de forma conservadora, los datos de la tomografía computarizada pueden persistir cinco a seis meses. ${ }^{2,16}$

La resonancia magnética revela datos de lesiones focales, con imágenes hipointensas que corresponden a grasa, que se muestran como anillo. Es un excelente método, pero está limitado por su costo y su menor disponibilidad. ${ }^{20,17,5}$
Estos estudios paraclínicos son útiles para descartar el resto de patologías con las que se debe realizar el diagnóstico diferencial: apendicitis, diverticulitis, infarto omental, paniculitis mesentérica, neoplasias o traumatismos. ${ }^{4,7}$

El tratamiento es conservador, con el uso de antiinflamatorios. El proceso se autolimita en menos de diez días. ${ }^{21,22,1}$

El tratamiento quirúrgico está reservado para los casos en los que fracasa el tratamiento médico o cuando hay complicaciones; que son raras; una de ellas puede ser la compresión extrínseca del colon adyacente, que ocasiona obstrucción intestinal; otras más raras, son la invaginación o la perforación intestinal. ${ }^{11,21}$

El pronóstico con terapia simple y conservadora es excelente y lleva a la rápida recuperación. ${ }^{15,22,4}$.

El conocimiento de esta entidad y sus hallazgos en las imágenes es de suma importancia, para no confundirla con otros procesos.

La apendicitis epiplóica puede semejar otros procesos inflamatorios abdominales.

La importancia de este caso clínico, es alertar al médico y a los cirujanos pediatras, de la existencia de esta patología, que por su rareza y manifestaciones inespecíficas, presentan un reto diagnóstico. Se resalta la importancia de los estudios de laboratorio y gabinete, pensando en las diferentes causas que pueden condicionar un cuadro de abdomen agudo quirúrgico.

\section{BIBLIOGRAFÍA}

1. Sand M, Gelos M, Bechara F, Sand D, Wiese T, Steinstraesser L, Mann B. Epiploic appendagitis-clinical characteristics of an uncommon surgical diagnosis. BMC Surg. 2007;7(11):1-7.

2. Mayank J, Shashi K, Bimalendu S, Om T. Torsion of the epiploic appendix: An unusual cause of acute abdomen. J Min Access Surg. 2007;1(3):320-8.

3. Vipul G, Kumar S. Appendicitis epiploicae: An unusual cause of acute abdomen in children. Pediatr Neonat Surg. 2008;13(2):83-5.

4. Cembellín F, Pardo P, Fernández P. Apendicitis epiplóica como causa de dolor agudo en fosa iliaca izquierda: a propósito de un caso. Emergencias. 2007;19:39-41.

5. Friedlich M, Papadatos D. Acute appendagitis as a cause of right lower quadrant pain. Can J Surg. 2006;49(3):215-17.

6. Patel V, Rao A, Reginald W, Srinivasan R, Fortson J, Weaver W. Cecal epiploic appendagitis: A diagnostic and therapeutic dilemma. Am Surg. 2007;73(8):828-30.

7. Rao P, Wittenberg J, Lawrason N. Primary epiploic appendagitis: Evolutionary changes in CT appearance. Radiology. 2007;204(3):713-17.

8. Hurreiz $\mathrm{H}$, Madavo $\mathrm{C}$. Torsion of an epiploic appendix mimic- 
king acute appendicitis. Saudi Med J. 2005;26(4):28-34.

9. Barbier C, Denny P, Pradoura J, Bui P, Rieger A, Bazin C. Radiologic aspects of infarction of the appendix epiploica. J Radiol. 1998;79(6):1479-85.

10. Hollerweger A, Macheiner $\mathrm{P}$, Hubner $\mathrm{E}$, Rettenbacher $\mathrm{T}$, Gritzmann N. Epiploic appendagitis: Sonographic findings in 28 cases. Ultraschall Med. 2002;23(8):239-44.

11. Pozo P, Lirón R, Pérez E, Soto A, Flores B, Perelló M, Martín $\mathrm{J}$, Moreno A, Aguayo J. Rotura de colon durante una colonoscopia secundaria a la torsión de un apéndice epiploico. Rev Esp Enferm Dig. 2006;98(10):789-98.

12. Varela C, Fuentes M, Rivadeneira R. Procesos inflamatorios del tejido adiposo intraabdominal. Causa no quirúrgica de dolor abdominal agudo: hallazgos en tomografía computada. Rev Chilena Radiol. 2004;10(1):28-34.

13. Sagayo D. Torsión de un apéndice epiploico. Reporte de 2 casos: características ecográficas y tomográficas. Acta Méd Costarricense. 2002;44(1):34-5.

14. Molinares B, Castrillón G, Restrepo R. Apendicitis epiploica. Reporte de cuatro casos. Rev Colomb Cir. 2006;21(3):196200.
15. O'Rourke R, Saito J, Albanese C. Laparoscopic diagnosis and resection of pediatric appendicitis epiploicae: Case report and literature review. Pediatr Endosurg. 2001;5(3):319-25.

16. Castro F, Santos A, García P, Díez J. Epiploic appendicitis. Rev Esp de Enferm Dig. 2006;98(2):140-2.

17. Núñez C, Raimunde E, Álvarez S, Olcoz J. Epiploitis aguda: una causa inusual de abdomen agudo médico. An Med Interna. 2006;23(10):507-10.

18. DíezA, Benejam F. Perforación divertículo vesical por apéndice epiploico. Actas Urol Españolas. 2007;31(4):429-32.

19. Kantarci M, Duran C, Sirvanci M. Gastrointestinal: Epiploic appendagitis. J Gastroenterol Hepatol. 2005;20(482):482-5.

20. Seehaus A, Ocantos J. Paciente con dolor abdominal. Acta Gastroenterol Latinoam 2007;37(1):70-3.

21. Vriesman A, Cobben L, Coerkamp E, Puylaert J. Epiploic appendagitis: MR appearance. Clin Radiol Extra. 2002;57(3):3-6.

22. Horvath E, Majlis S, Seguel S, Whittle C, Mackinnon J, Niedmann J, Baldassare G, González P. Apendicitis epiploica primaria: diagnóstico clínico y radiológico. Rev. Med Chile. 2000;128(6):1-10.

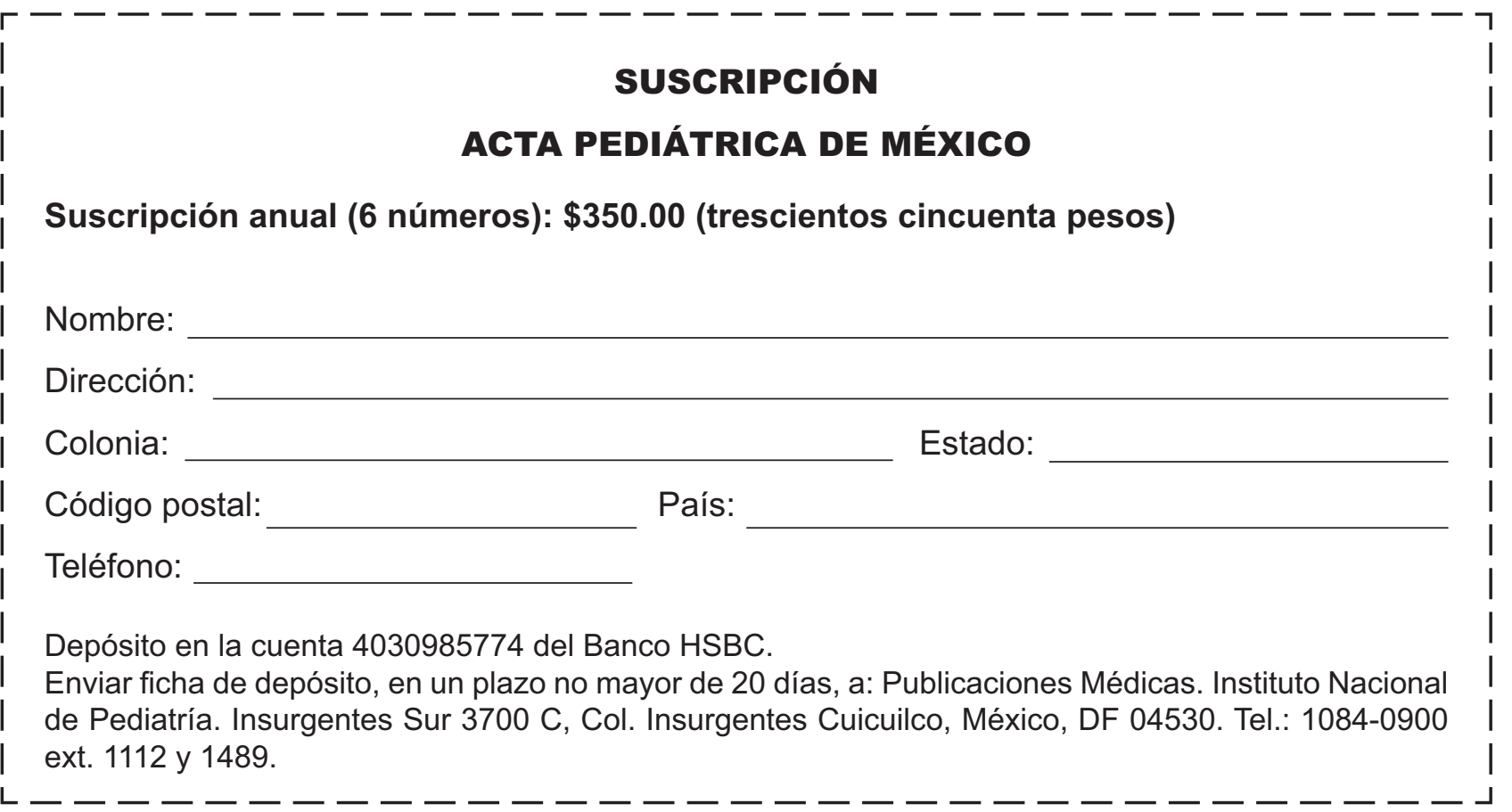

